\title{
Direct Modeling of Scintillator Thickness for Optimal Light Output and Spatial Resolution
}

\author{
S. E. Mitchell, A. Luttman, M. Fowler, K. T. Joyce \\ National Security Technologies, LLC \\ North Las Vegas, NV, 89030 \\ Email: mitchese@nv.doe.gov, luttmaab@nv.doe.gov, \\ michael.fowler22@gmail.com, kevin.t.joyce@gmail.com
}

\begin{abstract}
It is common in $\mathrm{x}$-ray radiography to use scintillators (e.g., BGO or LSO) to convert x-rays to visible light, which is then recorded by an imaging system. The response of the scintillator depends fundamentally on its thickness, with respect to both its visible light emittance and its spatial resolution. This is important for optimizing light output, signal to noise ratio, or optical response time. Given that it is often cost-prohibitive to procure a variety of scintillator samples and empirically test the performance, it is essential to be able to model and accurately simulate the performance of a scintillator with respect to thickness and other properties, and a direct way of doing this is using Monte Carlo-based radiation transport codes. Such simulations can be expensive in terms of computational time, and the codes are not easily obtained. In this work we first show such simulations, and demonstrate that there is a natural trade-off between light output of a scintillator and its spatial resolution. We then derive a first-principles model that accurately approximates the light output, using straightforward calculations that can be performed quickly with any basic computing software. We compare the results to those obtained from Monte Carlo simulations and show that our simplified model can be used to analyze the tradeoff between emittance and resolution nearly as well as using a full-scale radiation transport code.
\end{abstract}

\section{INTRODUCTION}

In x-ray and radiography applications, a scintillator is often used as a light converter; a device that absorbs an input photon at some particular energy and emits a photon at some other energy. In practice, the input photons are typically high x-ray energies, whereas the output photon energy is essentially constant, typically visible, and nearly transparent with respect to the scintillating crystal. The benefit of using a scintillator is that it allows one to infer the density of an object through x-ray photon attenuation without needing an $\mathrm{x}$-ray detector, by measuring the visible light emitted by the scintillator and collecting it with a standard CCD camera. In order for the scintillator to be useful, however, it must output sufficiently intense visible light, relative to the intensity of the input $\mathrm{x}$-rays, and it must have a spatial resolution (response function) that is sufficiently fine. Both the resolution and the visible emittance of a scintillator depend fundamentally on its thickness, so, when choosing a scintillator for a given application, the thickness of the scintillator is a primary concern. Since thicker scintillators emit higher intensity light (generally speaking) but also have coarser spatial resolution, there is a tradeoff between these two important properties, and for each application an optimal thickness must be found. Of course one can test scintillator response for a variety of thicknesses [1], but purchasing and testing a variety of scintillator thicknesses is often cost-prohibitive. Thus it is desirable to model and simulate the performance of a scintillating material at a variety of thicknesses before purchasing, and in this work we analyze two methods for simulating the emittance and resolution of a scintillator as a function of thickness.

One natural approach to modeling a scintillator is to use a large-scale Monte Carlo-based radiation transport code. Several such codes exist and have been vetted over decades, giving extremely reliable results. Unfortunately, Monte Carlo calculations can be very computationally expensive, and the codes themselves are not easily obtained by the general public. Thus it is of interest to generate simplified, first-principles models for calculating emittance and resolution of a scintillator that can be easily implemented on generally available systems and that give accurate results in a short amount of time. Section II presents results of a Monte Carlo simulation of a Saint Gobain PreludeTM 420 [2], which is $\mathrm{Lu}_{1.8} \mathrm{Y}_{.2} \mathrm{SiO}_{5}$ :Ce at a density of $7.2 \mathrm{~g} / \mathrm{cc}$ (hereafter referred to simply as LSO). In Section III, we present a simplified model for estimating scintillator emittance, and Section IV compares the results and shows that the simplified model can be used to find approximately optimal scintillator thickness for certain applications.

\section{Modeling Scintillators With Monte CARlo CODES}

MCNPX [3] is a well-vetted radiation transport code developed at Los Alamos National Laboratory. It contains physical models for particle transport as well as large databases of material properties with interaction cross-sections. Using this information it computes trajectories (called "histories") of individual particles through the computational domain, integrating energy deposition at each grid point and calculating statistics associated with the simulation. Given a sufficiently large number of particle histories, the performance of a system can be well approximated.

In this case, we used MCNPX to simulate the response of an LSO scintillator due to an impinging energetic source photon beam. Fig. 1 shows the deposition of $x$-ray photons at each point in the scintillator, given a pencil beam of source photons emitted upward into the bottom face of the scintillator crystal. Two million particles are simulated in these plots. The simulation is performed for several source energies, and the 2D, integrated energy deposition profiles are shown in Fig. 2 for an LSO scintillator that is $10 \mathrm{~cm}$ thick (vertical scale) and $2 \mathrm{~cm}$ wide (horizontal scale). As is expected, the greatest deposition is along the central axis where the 


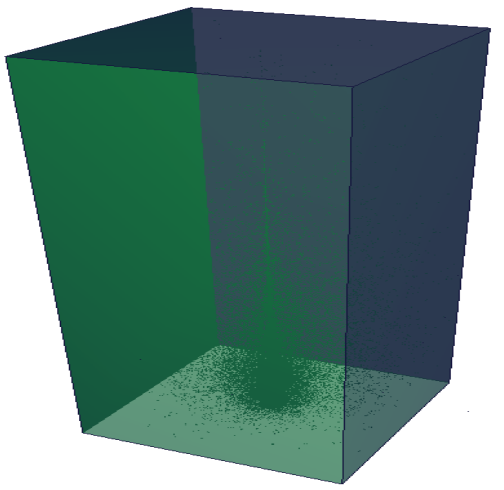

Fig. 1. 3-D Particle tracks of $0.5 \mathrm{MeV}$ source particle energy deposition in YLSO scintillator. The X-ray beam penetrates the scintillator in the center of the bottom face of the cube, and each point inside in the cube represents an $\mathrm{x}$-ray being absorbed by the material.

pencil beam penetrates the crystal, with deposition trailing off exponentially in the $z$ axis (vertical) and even faster in the $y$ axis (horizontally out from the center). For $0.5 \mathrm{MeV}$-rays (left-most plot), nearly all $\mathrm{x}$-rays have been deposited within $5 \mathrm{~cm}$, resulting in dark blue at the top of the plot where little energy is being deposited. For $2 \mathrm{MeV}$, there are $\mathrm{x}$-ray photons being deposited all the way through the scintillator.
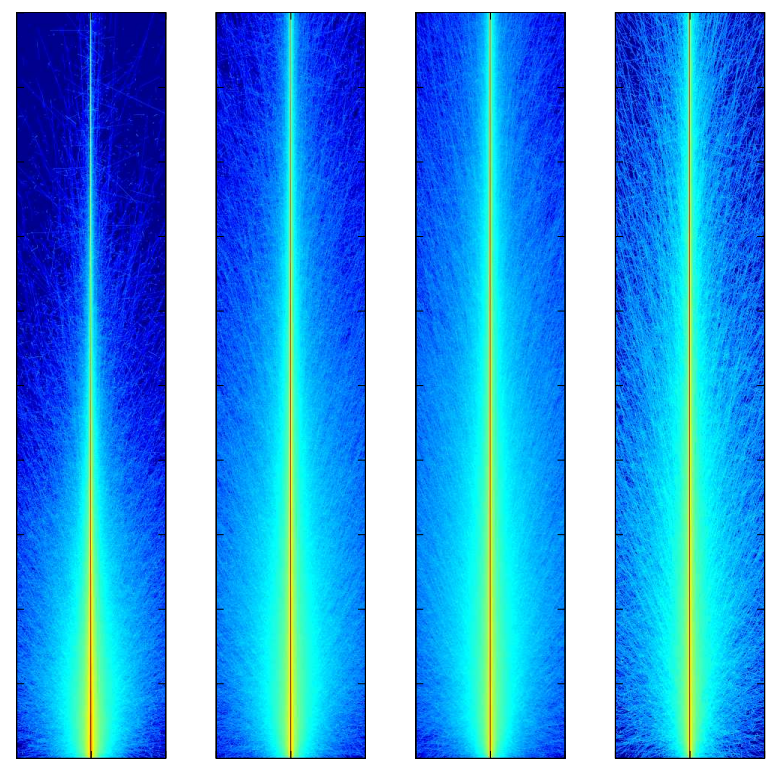

Fig. 2. [Left to Right] The logarithm of 2-D energy deposition for $0.5,1$, 1.5 , and $2 \mathrm{MeV}$ source energies. The $z$ (vertical) coordinates are from 0 to $10 \mathrm{~cm}$, and the $y$ coordinates (horizontal) are from $-1 \mathrm{~cm}$ to $1 \mathrm{~cm}$, with the pencil beam impinging on the crystal at the point $(0,0)$.

There are two quantities of interest. The first is the number of visible photons emitted, and it is commonly assumed that the number of photons emitted at each point is proportional to the number of photons absorbed at that point, depending on the energy of the input photons. We denote this scale factor by $\Phi_{\text {Scint }}(E)$, and for LSO, $\Phi_{\text {Scint }}(E) \approx 32000$ for the input energies of interest and that value is used throughout this work. In order that the visible photons contribute to
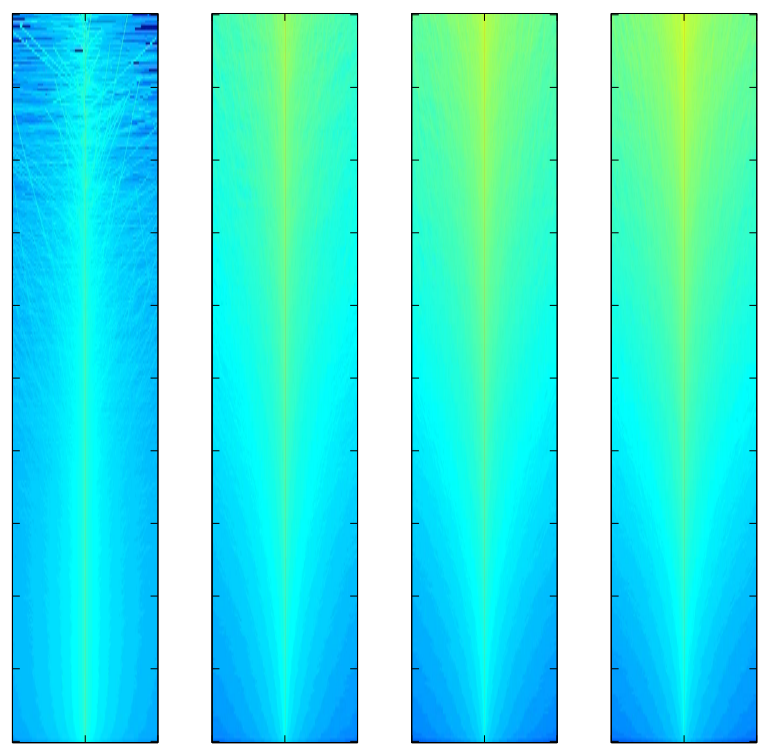

Fig. 3. Relative photon emittance for $0.5,1.0,1.5$, and $2.0 \mathrm{MeV}$ (left to right) with a thickness of $10 \mathrm{~cm}$. The primary emittance in the $10 \mathrm{~cm}$ scintillator occurs near to the top, i.e. near to the $10 \mathrm{~cm}$ point, which is inefficient, since most deposition occurs near 0 (at the bottom).

the scintillator response, they must survive self-absorption in the material during their travel, so the light output of the scintillator calculated by the Monte Carlo simulation is related to the energy deposition via

$$
I_{\mathrm{MC}}(L, E)=\Phi_{\text {Scint }}(E) \int_{-y_{0}}^{y_{0}} \int_{0}^{L} D(y, z, E) e^{-\left(\frac{L-z}{\lambda_{p}}\right)} d z d y,
$$

where $L$ is the thickness of the scintillator, $\lambda_{p}$ is the mean free path length of the emitted photons in LSO $\left(\lambda_{p}=1.2\right.$ cm for LSO, [4], [5], [6]), and $D(E, y, z)$ is the total energy deposition at a scintillator point $(y, z)$ for the given input photon energy $E$ (the "data" computed by the Monte Carlo code), $2 y_{0}$ is the width of the scintillator, and $I_{\mathrm{MC}}$ is the light output of the scintillator. Note that Fig. 2 shows the logarithm of deposition, which means that nearly all x-ray photons deposited and nearly all visible photons emitted are interacting along the central vertical axis. This suggests that the $y$ dependences of light output and maximum light output are negligible. Fig. 4 shows the visible light output as a function of scintillator thickness for $0.5,1,1.5,2$, and $2.5 \mathrm{MeV}$ input photon energies integrated over the entire scintillator (dashed curves). It shows the same quantities, integrated only over the central axis of the scintillator (solid curves), and the thicknesses that maximize light output over the entire scintillator and the thicknesses that maximize light output over just the central axis are equal (to less than $0.01 \%$ ). Thus, from here on, we only analyze light output along the central axis. As can be seen in Fig. 4, the maximum light output increases with energy, as does the thickness at which light output is maximized. 


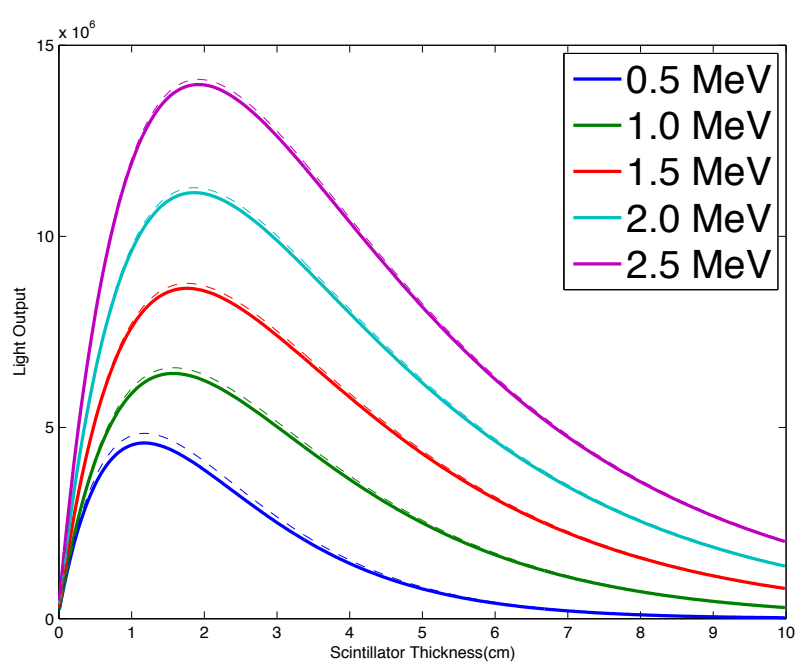

Fig. 4. Light output as a function of scintillator thickness for specific input photon energies (measured in number of particles on the vertical axis). The dotted curves give total light output integrated over the entire scintillator, and the solid curves show light output corresponding just to the central axis.
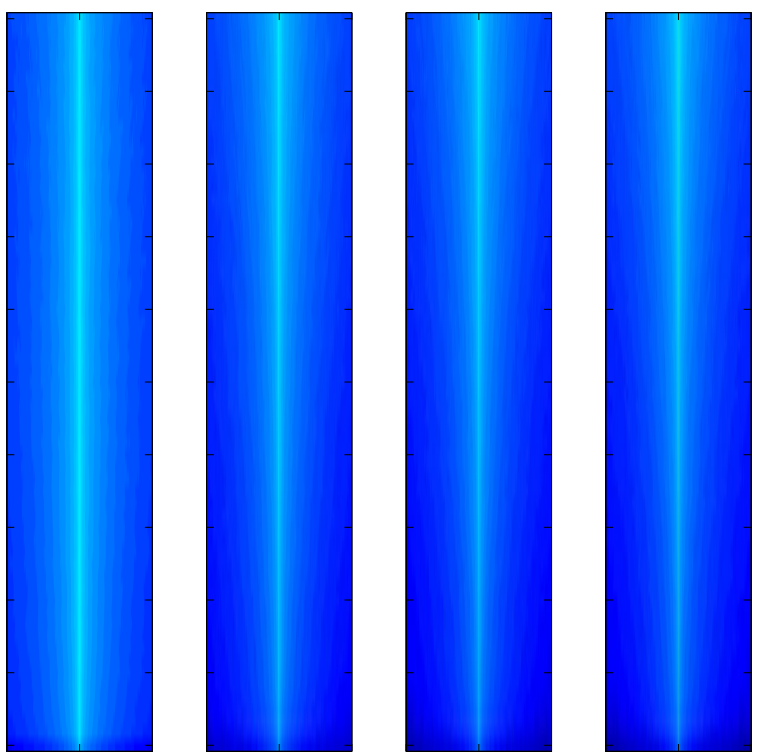

Fig. 5. Relative photon emittance for $0.5,1.0,1.5$, and $2.0 \mathrm{MeV}$ (left to right) with a thickness of $2 \mathrm{~cm}$. Since the maximum light output occurs for thicknesses less than $2 \mathrm{~cm}$, this calculation is more applicable than that in Fig. 3. For this thickness, emittance is more uniform in $z$.

The second quantity of interest is the scintillator spatial resolution, and this can also be estimated from the Monte Carlo simulations. If one computes the spatial resolution of the scintillator as a radially-symmetric point spread function that gets convolved into the total radiographic system response, then the line spread function (LSF) of the scintillator is the
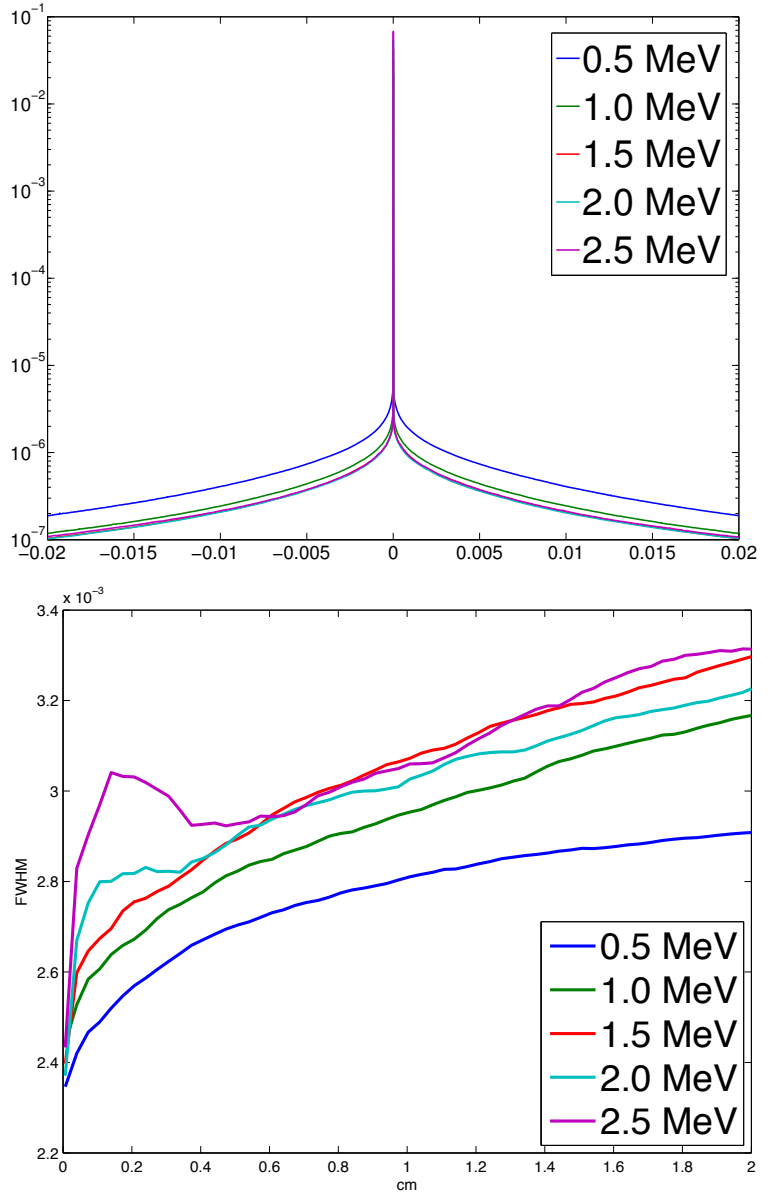

Fig. 6. (Top) An approximate LSF for the scintillator response is computed by calculating the integrals along parallel lines (vertical lines when visualized with Fig. 2) in the MCNPX results, assuming a $2 \mathrm{~cm}$ scintillator. (Bottom) For each LSF we compute the full-width, half-maximum value as a function of the thickness of the scintillator (vertical units are also $\mathrm{cm}$ ). The FWHM increases with input energy and with thicknes

Abel transform of the PSF. The Abel transform is computed as integrals along parallel lines, so the MCNPX data $D(E, y, z)$, multiplied by the decay factor $\exp \left(\frac{-(L-z)}{\lambda_{p}}\right)$, can be integrated along the vertical lines to give an approximation to the LSF. A common metric for quantifying spatial resolution is the full-width, half-maximum (FWHM) of the PSF or LSF. The LSF, as a function of thickness, is shown for each input energy in Fig. 6 (top), and Fig. 6 (bottom) shows the FWHM of the LSF for each input energy as a function of scintillator thickness. The curves are not smooth - and not even monotonic - because they are computed from the MCNP simulations, which have a stochastic component.

As can be seen from the figure, the FWHM of the LSF for $0.5 \mathrm{MeV}$ varies from approximately 2.3 to approximately $2.9\left(\times 10^{-3} \mathrm{~cm}\right)$ over a thickness range from 0 to $1.5 \mathrm{~cm}$, a variation of about $25 \%$. The results are similar for the other energies, with the variation for $2.5 \mathrm{MeV}$ being about $30 \%$. Since $2 \mathrm{~cm}$ is the maximum thickness to be accounted for in terms of light output, one should now choose a thickness between 0 and $2 \mathrm{~cm}$, based on the desired resolution for the 
given application.

\section{A SIMPLIFIED FIRST-PRINCIPLES MODEL}

The analysis developed in the previous section is helpful for optimizing scintillator thickness for a given application, assuming that one has access to radiation transport codes and significant computing power. Without such resources, it is desirable to have a simple, first-principles model for approximating scintillator emittance and spatial resolution as a function of thickness, and in this section we present such a model.

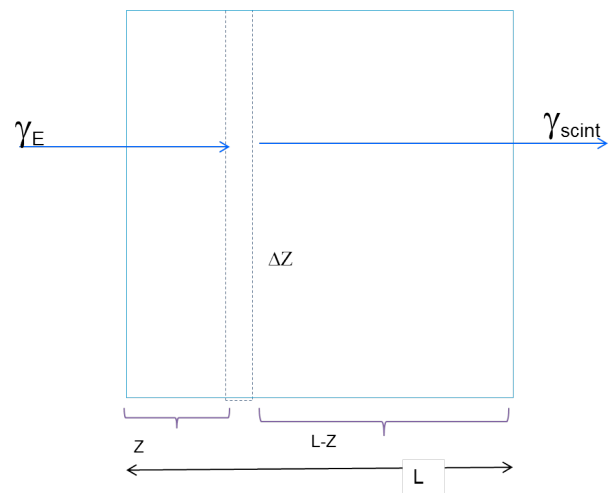

Fig. 7. A schematic of a photon interaction with the scintillator, where $\gamma_{\text {Scint }}(E)$ is the flux of a monoenergetic input source, $\gamma_{\text {Scint }}$ is the flux of visible photons out of a section of thickness $\Delta z$, and $L$ is the thickness of the scintillator.

In order for a visible photon to be emitted from the scintillator, an $\mathrm{x}$-ray must penetrate the scintillator to a given thickness $z$, it must be absorbed by the crystal in a small thickness $\Delta z$, then the emitted photon must survive the remaining distance to escape the crystal (see Fig. 7). Thus the probability of emittance along a single line in $z$ is approximately proportional to

$$
P_{\mathrm{emit}} \approx\left(e^{-\frac{z}{\lambda_{z}}}\right)\left(1-e^{-\frac{\Delta z}{\lambda_{z}^{\prime}}}\right)\left(e^{-\frac{L-z}{\lambda_{p}}}\right)
$$

The values for $\lambda_{z}(E)$ are determined from mass attenuation coefficient values for a particular scintillator. By determining the appropriate partial mass fraction for each of the constituent materials (Table 1), the following may be used to compute the total equivalent mass attenuation coefficient for the scintillator:

$$
\frac{\mu}{\rho}=\sum_{i} w_{i}\left(\frac{\mu}{\rho}\right)_{i}
$$

where $w_{i}$ is the fraction by weight of the $i^{\text {th }}$ atomic constituent, and the $(\mu / \rho)_{i}$ values are from Table 3 of the NIST physical reference database [7]. Finally, the $(\mu / \rho)_{i}$ values are multiplied by the mass density of $7.2 \mathrm{~g} / \mathrm{cc}$ to yield $\frac{1}{\lambda_{z}(E)}$, and the resulting reciprocal path length coefficient values for the scintillator is shown in Table I. The values for $\lambda_{z}^{\prime}(E)$ are similarly computed using the reciprocal energy absorption coefficient, $\mu_{E} \rho$, for scintillator material.

In order to use this to develop a model for light output and spatial resolution, we must also factor in the probability of deposition with respect to $y$. Here we make the simplifying
TABLE I. MASS FRACTION CALCULATION FOR THE SAINT GobAIN 420 LSO.

\begin{tabular}{|c|c|c|c|c|}
\hline Element & A & \# moles & M (g) & wt. fraction \\
\hline $\mathrm{Lu}$ & 176 & 1.8 & 316.8 & 0.714 \\
$\mathrm{Y}$ & 89 & 0.2 & 17.8 & 0.040 \\
$\mathrm{Si}$ & 28 & 1 & 28 & 0.063 \\
$\mathrm{O}$ & 16 & 5 & 80 & 0.180 \\
$\mathrm{Ce}$ & 140 & 0.01 & 1.4 & 0.003 \\
\hline \multicolumn{4}{|c|}{ Total M (g): } \\
\hline
\end{tabular}

assumption that the probability in $y$ is decoupled from the probability in $z$, which gives

$$
P_{\mathrm{emit}} \approx\left(e^{-\frac{z}{\lambda_{z}(E)}}\right)\left(1-e^{-\frac{\Delta z}{\lambda_{z}^{\prime}(E)}}\right)\left(e^{-\frac{L-z}{\lambda_{p}}}\right) D_{2}(y, E)
$$

where $D_{2}(y)$ is the probability density of deposition along the $y$ axis.

Letting $\Delta z \rightarrow 0$, gives the approximation

$$
1-e^{-\frac{\Delta z}{\lambda_{z}^{\prime}}} \approx \frac{d z}{\lambda_{z}^{\prime}}
$$

so integrating over the scintillator and multiplying by the photon conversion factor $\Phi_{\text {Scint }}$ results in a light output model of the form

$$
\begin{aligned}
I_{\text {Model }}(L, E)=\Phi_{\text {Scint }}(E) & \int_{-y_{0}}^{y_{0}} \int_{0}^{L} \frac{1}{\lambda_{z}^{\prime}(E)} D_{2}(y, E) \\
& \left(e^{-\frac{z}{\lambda z(E)}}\right)\left(e^{-\frac{L-z}{\lambda_{p}}}\right) d z d y
\end{aligned}
$$

which simplifies to

$$
\begin{aligned}
I_{\text {Model }}(L, E)= & \frac{\Phi_{\text {Scint }}(E)}{\lambda_{z}^{\prime}(E)}\left(e^{-\frac{L}{\lambda_{z}(E)}}-e^{-\frac{L}{\lambda_{p}}}\right) \\
& \frac{\lambda_{z}(E) \lambda_{p}}{\lambda_{z}(E)-\lambda_{p}} \int_{-y_{0}}^{y_{0}} D_{2}(y, E) d y . \\
= & \Psi(E)\left(e^{-\frac{L}{\lambda_{z}(E)}}-e^{-\frac{L}{\lambda_{p}}}\right)
\end{aligned}
$$

where

$$
\Psi(E)=\frac{\Phi_{\text {Scint }}(E)}{\lambda_{z}^{\prime}(E)}\left(\frac{\lambda_{z}(E) \lambda_{p}}{\lambda_{z}(E)-\lambda_{p}}\right) \int_{-y_{0}}^{y_{0}} D_{2}(y, E) d y
$$

is independent of $L$. Note that the dependance on $y$ contributes only a scale factor to the light output and does not impact the thickness at which output is maximized. This is in agreement with the MCNPX simulations, which also showed that the $y$ dependence did not impact the maximizing thickness.

Fig. 8 shows plots of the visible light emittance for an LSO scintillator as a function of thickness, using (3), for $0.5,1$, $1.5,2$, and $2.5 \mathrm{MeV}$ input source photons. The vertical scale in the figure is determined only up to an energy-dependent scale factor, but the shapes of the curves as a function of $L$ are correct. In particular, the plot correctly represents the thicknesses that correspond to the maximum visible light emittance, for the given energies. 


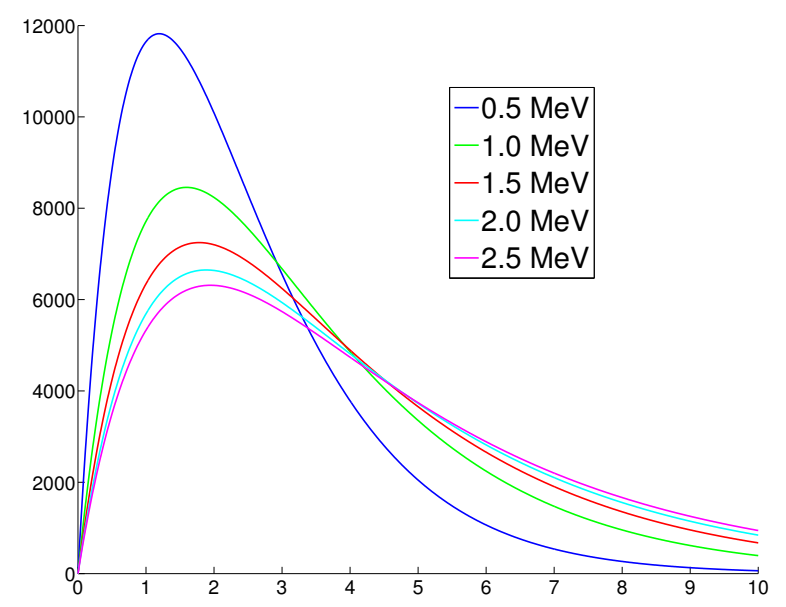

Fig. 8. Light output from model, without accounting for the energydependence of $D_{2}(y, E)$. The plots are missing an energy-dependent scale factor, so only the shape of the curves and the points at which light output are maximized are relevant.

\section{COMPARISON OF RESUltS}

The primary results of the Monte Carlo simulations presented in Section II and the first-principles model presented in Section III are shown in Table II. As can be seen, the two methods agree, differing by less than $3 \%$ for each of the 5 input energies analyzed, demonstrating that the first-principles model can accurately approximate the thickness of a scintillator that maximizes its light output. This gives an upper bound on the thickness, and then the desired spatial resolution for the given application dictates if a smaller thickness should be used.

TABLE II. SCINTILLATOR THICKNESS CORRESPONDING TO MAXIMUM COMPUTED LIGHT OUTPUT FOR AN LSO SCINTILLATOR OF WIDTH 2 CM AND LENGTH 10 CM FOR $0.5,1,1.5$, AND 2 MEV INPUT PHOTON ENERGIES FOR MONTE CARLO SIMULATIONS AND SIMPLIFIED MODEL.

\begin{tabular}{|c|c|c|}
\hline Input Energy & $\begin{array}{c}I_{\mathrm{MC}} \text { Optimal Thickness } \\
(\mathrm{cm})\end{array}$ & $\begin{array}{c}I_{\text {Model }} \text { Optimal Thickness } \\
(\mathrm{cm})\end{array}$ \\
\hline $0.5 \mathrm{MeV}$ & 1.1745 & 1.20 \\
$1.0 \mathrm{MeV}$ & 1.5749 & 1.61 \\
$1.5 \mathrm{MeV}$ & 1.7751 & 1.79 \\
$2.0 \mathrm{MeV}$ & 1.8752 & 1.90 \\
$2.5 \mathrm{MeV}$ & 1.9086 & 1.96 \\
\hline
\end{tabular}

\section{ACKNOWLEDGMENT}

This manuscript has been authored by National Security Technologies, LLC, under Contract No. DE-AC5206NA25946 with the U.S. Department of Energy and supported by the Site Directed Research and Development program. The United States Government retains and the publisher, by accepting the article for publication, acknowledges that the United States Government retains a non-exclusive, paid-up, irrevocable, world-wide license to publish or reproduce the published form of this manuscript, or allow others to do so, for United States Government purposes. DOE/NV/25946-1809

\section{REFERENCES}

[1] S. Lutz and S. Baker, "Scintillator evaluation for high-energy X-ray diagnostics," September 2001, DOE/NV/11718-653.

[2] Saint Gobain Crystals, Scintillation Products, http://www.detectors.saintbain.com/PreLude420.aspx.
[3] J. F. Briesmeister, ed., "MCNP - A General Monte Carlo N-Particle Transport Code, Version 4C," Los Alamos National Laboratory report LA-13709-M (March 2000).

[4] Particle Data Group, Section 28.3, "Inorganic Scintillator: Reviews, Tables, and Plots" J. Phys. G: Nucl. Part. Phys., 33 (2006) 275.

[5] J. M. Chen, L. Y. Zhang and R. Y. Zhu, "Large size LYSO crystals for future high energy physics experiments," IEEE Trans. Nucl. Sci., 52 (2005) $3133 i ̈ ; 3140$.

[6] J. M. Chen, R. H. Mao, L. Y. Zhang, and R. Y. Zhu, "Large size LSO and LYSO crystal for future high energy physics experiments," IEEE Trans. Nucl. Sci., 54 (2007) $718 \ddot{i} £_{7} 724$.

[7] National Institute of Standards and Technology, Physical Reference Data, "X-Ray Mass Attenuation Coefficients," http://physics.nist.gov/PhysRefData/XrayMassCoef/chap2.html. 